\title{
Clinical impact of blood cultures taken in the emergency department
}

\author{
Anne-Maree Kelly
}

\begin{abstract}
Objectives-(1) To establish the proportion of blood cultures that yield a positive microbiological culture. (2) To determine what proportion of blood cultures taken in the emergency department impact on patient management. (3) To develop guidelines for the appropriate ordering of blood cultures from patients in the emergency department (ED).

Methods-Retrospective review of all blood cultures taken in the ED of a metropolitan teaching hospital between 26 September 1995 and 30 June 1996.

Results-One thousand and sixty two blood cultures were taken from patients in the ED during the study period. Ninety two $(9 \%)$ returned a positive microbiological culture. Of these, $52(5 \%)$ were "true" positives and $18(1.6 \%)$ resulted in changes in management.

Conclusions-Blood cultures taken in the ED rarely yield positive cultures. Only $1.6 \%$ of blood cultures taken in the ED impact on management of patients. Simple strategies could reduce the number of blood cultures ordered with little prospect of patient compromise.

( $($ Accid Emerg Med 1998;15:254-256)
\end{abstract}

Keywords: blood culture; sepsis; tests

Blood cultures are commonly part of the investigation of patients with suspected infective processes. Their use is recommended by major medical texts for the investigation of a wide variety of potential diagnoses. Despite this, there are no published studies that confirm their utility and/or clinical impact.

The indications for obtaining blood cultures from patients in the emergency department (ED) are even less clear. There is no clinical impact data specific to the ED practice setting and attempts to encourage informed use of this test are few. ${ }^{1}$ It is the author's opinion that blood cultures are more often ordered "because the patient has a fever" or "the admitting registrar will want them" than for any expectation of clinical impact. In the current economic climate this is difficult to justify, particularly as this test carries some risk to staff in the form of needlestick injury and blood borne infection.

In recent years, a small number of papers have been published looking at the clinical utility of blood cultures as a test. ${ }^{2-5}$ These, however, are difficult to extrapolate to the ED setting as they have either focused on hospital inpatients, patients in intensive care settings, or have "worked backwards" from discharge diagnosis.

This study was conducted to clarify some of the issues surrounding the ordering of blood cultures from the ED. Its aims were: (1) To establish the proportion of blood cultures that yield a positive microbiological culture. (2) To determine what proportion of blood cultures taken in the ED impact on patient management. (3) To develop guidelines for the appropriate ordering of blood cultures from patients in the ED.

\section{Methods}

Blood cultures ordered from the ED of Western Hospital Footscray for the period 26 September 1995 to 30 June 1996 were identified using the ED's computerised data collection system. Western Hospital Footscray has an annual census of approximately 35000 adult patients with an admission rate of $30 \%$. Paediatric patients are not treated at this department.

The following data were collected for each test: the patient's unit record number, the ED diagnosis, the result of the blood culture, and whether the patient was admitted to hospital for treatment. In those cases where a positive culture was returned, the patient's medical record was reviewed to attempt to establish whether the culture was a "true" or "false" positive. A true positive culture was defined as one where the organism grown was considered by the treating clinician or microbiologist to be responsible for the clinical illness. Additional data collected from the record included whether treatment was changed due to the positive culture and whether there were patient factors putting the patient in a high risk group for disseminated or atypical infection, such as diabetes mellitus, cancer on chemotherapy, or with long term indwelling lines. When there was any doubt about the status of a result as "true" or "false" positive, it was classified as "true" positive.

Data were analysed according to ED diagnosis and disposal to determine the potential impact of selective test ordering.

\section{Results}

One thousand and sixty two blood cultures were taken from patients in the ED during the study period. Ninety two $(9 \%)$ returned a positive microbiological culture. Of these, 52 $(5 \%)$ were "true" positives and $18(1.6 \%)$ resulted in changes in management. The results for the major classifications of ED diagnosis are summarised in table 1 . Height of fever, white cell count, and neutrophil count 
Table 1 Rate of positive blood culture and clinical impact for selected diagnoses

\begin{tabular}{lll}
\hline Clinical diagnosis in ED & $\begin{array}{l}\text { \% "True" } \\
\text { positive rate } \\
\text { (total cases) }\end{array}$ & $\begin{array}{l}\text { Where blood } \\
\text { culture altered } \\
\text { treatment (\%) }\end{array}$ \\
\hline Pneumonia & $5(260)$ & 1 \\
Pyelonephritis & $7(44)$ & 0 \\
Cholangitis & $40(5)$ & 0 \\
Cancer with fever & $12(26)$ & 8 \\
Clinical septicaemia & $15(33)$ & 9 \\
Abdominal pain with fever & $3(29)$ & 0 \\
Cardiac failure & $0(21)$ & 0 \\
COAD & $0(70)$ & 0 \\
Infective arthritis/ & $10(9)$ & 0 \\
$\quad$ osteomyelitis & $2(82)$ & 0 \\
Cellulitis & & \\
\hline
\end{tabular}

COAD = chronic obstructive airway disease.

Table 2 Characteristics of the 18 patients where positive blood cultures resulted in changes in management

\begin{tabular}{ll}
\hline Characteristics & No \\
\hline Cancer on chemotherapy with fever & 2 \\
Diabetic, no direct specimen available & 2 \\
Long term indwelling lines with fever & 2 \\
Clinical endocarditis, diagnosed in ED & 2 \\
Persistent fever of unknown origin & 3 \\
Focal infection: clinically obvious & 4 \\
Other (1 pneumonia, 1 typhoid, 1 uncertain origin) & 3 \\
\hline
\end{tabular}

were not helpful in predicting the likelihood of positive blood culture.

The features of the 18 positive cultures that resulted in changes in management are summarised in table 2 . One of the 18 , with an eventual diagnosis of endocarditis, had been discharged from the ED and was recalled for treatment. Two of the focal infections had a microbiological identification made from a direct specimen before the results of blood culture were available, however it was unclear which result resulted in management change.

The potential impact of selective test ordering was considerable. Refraining from taking blood cultures in cases where a direct specimen was available would have reduced the number of tests ordered by $18 \%$. A further $14 \%$ reduction could have been achieved if blood cultures were not taken in patients who did not require hospital admission. An additional 9\% reduction was possible if this test was not performed initially for immunocompetent patients with "typical" infections such as cellulitis, orchitis, and dental infections. Further reduction, of the order of $20 \%$, was possible if blood cultures were not taken routinely in immunocompetent patients with community acquired pneumonia or patients with conditions associated with infections but not primarily infective such as asthma or cardiac failure (assumes $50 \%$ of cases had other indications for blood culture). Overall, selective ordering of blood cultures could result in a approximately $60 \%$ reduction in the number of tests ordered.

\section{Discussion}

Blood cultures are a commonly ordered test in EDs. There are a number of reasons for this behaviour. Habit is a major factor. Hospital based training reinforces the almost pavlovian response that if a patient has fever a blood culture should be taken. Also, there is a perception among ED staff that blood cultures should be taken because the inpatient unit will require them. The underlying assumptions that the inpatient unit is always right and that the blood cultures will alter management have yet to be proved! In addition, there is a lack of appreciation of the sensitivity and specificity of blood cultures as a test. Studies have shown that in the presence of known major bacterial infection, blood cultures are only positive in up to $20 \%$ of cases. ${ }^{56}$ In most of these studies, multiple blood cultures have been taken, so the sensitivity of an individual test is likely to be lower than this. The pathology of the disease and thus the likelihood of bacteraemia must also be considered. An additional factor is the prevalent fallacy that if blood cultures are not performed initially, "the boat has been missed". Recent experience in oncology would challenge this belief. And it should be remembered that the rate of false positive blood cultures is approximately $50 \%,{ }^{1}$ a figure confirmed by the findings of this study.

The results of this study show that blood cultures obtained from patients in the ED rarely yield a result that impacts on patient management. This is consistent with the findings of previous studies. ${ }^{2} 3$

Assuming an average cost of $A \$ 30$ per test, the current study shows that the cost per test that alters management is approximately A $\$ 1770$. This is consistent with Henke's finding of US\$ 1877 per test resulting in altered patient management. ${ }^{2}$

The clinical impact of blood cultures must be compared with the clinical impact and costs of more direct tests such as cerebrospinal fluid Gram stain, culture and latex agglutination, urine and joint fluid culture, and swabs from skin lesions. With respect to cerebrospinal fluid, the diagnostic yield of cerebrospinal fluid culture ranges from 48 to $100 \%$, depending on whether the patient has been pretreated with antibiotics, ${ }^{78}$ direct Gram stain has a yield of $74 \%,{ }^{8}$ and latex agglutination tests, $80 \% .{ }^{8}$ Used together these three tests have been reported to have a diagnostic yield of $92 \%{ }^{8}$ With respect to synovial fluid culture, cultures are positive in almost all cases of nongonococcal bacterial septic arthritis. ${ }^{9}$ In gonococcal disease, synovial fluid culture is positive in 25 to $50 \%$ of cases, however genital cultures increase diagnostic yield markedly. ${ }^{9}$ Regarding pyelonephritis, a positive urine culture is usually a defining criterion, so the sensitivity of urine culture approaches $100 \%$. Of note with respect to the clinical impact of blood cultures, in this study, all patients with clinical pyelonephritis and a positive blood cultures also had the same organism grown from their urine.

In this study, blood culture results were not matched to gold standards. Other than urine in pyelonephritis, the gold standards such as cerebrospinal fluid, joint aspirate, and sputum do not have $100 \%$ culture positive rates and as such are not true gold standards and in fact may be misleading if used for comparison. For other conditions like subacute bacterial endocarditis, gold standard material is not readily available.

In the ED the decisions to be made are whether to admit the patient and which antibi- 
Table 3 Guidelines for ordering blood cultures in the ED

Blood cultures should only be taken if

(1) After clinical assessment, the patient's illness is thought likely to be bacterial in origin and

(2) No more direct specimen for culture (for example, urine, wound swab, cerebrospinal fluid, joint aspirate, etc) is practical and

(3) The patient's condition is such that treatment as an inpatient is warranted

Blood cultures should not be taken routinely from stable, immunocompetent patients with common or typical infections such as cellulitis, orchitis, and community acquired pneumonia

otics (if any) to use. As the results of blood cultures are not available for several days, they have no impact on ED decision making. This rests on clinical assessment and more immediately available tests.

The ED diagnosis and disposal analysis suggests that the institution of some simple guidelines could markedly reduce the number of tests ordered and hence reduce investigation costs. For ease of use, this analysis has been simplified into the guidelines shown in table 3 . It is estimated that the use of these guidelines would result in a $40 \%$ reduction in the number of blood cultures ordered that equates to a saving of approximately $\mathrm{A} \$ 18000$ per annum for the study ED. This saving could be further increased if it was decided not to take blood cultures from selected patients with community acquired pneumonia.

It could be suggested that reducing the number of blood cultures taken would reduce the pool of microbiological data on which we base clinical decisions, particularly about the choice of antibiotic used as first line treatment. This is unlikely to apply because if appropriate direct specimens are taken (with their higher culture yield) the pool of microbiological data should be undiminished.

The finding that blood cultures taken in the ED have very low clinical impact should raise the same question about other tests used "routinely" in EDs such as abdominal radiography for abdominal pain and full blood examinations for a wide variety of illnesses. With significant budgetary constraints, we are challenged to understand the performance charac- teristics of the tests we use and their potential clinical impact in order to use them effectively and efficiently.

One of the biggest challenges to change in patterns of test ordering is changing attitudes outside the ED. This will require sharing of clinical impact data with inpatient clinicians and joint approaches to testing policy development.

\section{Conclusion}

Blood cultures taken in the ED rarely yield positive cultures. Only $1.6 \%$ of blood cultures taken in the ED impact on management of patients. Simple strategies could reduce the number of blood cultures ordered with little prospect of patient compromise.

1 Salluzzo R, Reilly K. The rational ordering of blood cultures in the emergency department. Qual Assur Util Rev 1991;6:28-31.

2 Henke PK, Polk HC Jr. Efficacy of blood cultures in the critically ill surgical patient. Surgery 1996;120:752-8.

3 Chendrasekhar A. Are routine blood cultures effective in the evaluation of patients clinically diagnosed to have nosocomial pneumonia? Am Surg 1996;62:373-6.

4 Clements H, Stephenson TJ. Blood culture is poor method of confirming pneumococcus as cause of childhood of confirming pneumococcus as cause

5 McMurray BR, Wrenn KD, Wright SW. Usefulness of blood cultures in pyelonephritis. Am J Emerg Med 1997;15:13740.

6 Baraff LJ. Blood culture in children with pneumonia. Ann Emerg Med 1996;27:774-6.

7 Bhisitkul DM, Hogan AE, Tanz RR. The role of bacteria antigen detection tests in the diagnosis of bacterial meningitis. Pediatr Emerg Care 1994;10:67-71.

8 Rodriguez G, Boehme C, Soto L, et al. Diagnosis of bacterial meningitis by latex agglutination tests. Rev Med Chil 1993;121:41-5.

9 Lorei JM. Synovial fluid analysis. In: Wolfson AB, Paris PM, eds. Diagnostic testing in emergency medicine. Philadelphia: WB Saunders, 1996. 